\title{
Engineered Nanomaterials and Human and Environmental Health: Research Strategies to Address Potential Risks
}

\author{
Jonathan M. Samet
}

Published online: 21 June 2014

(C) Springer International Publishing AG 2014

\begin{abstract}
Engineered nanomaterials have structured components less than 100 nanometers or $0.1 \mu \mathrm{m}$ in greatest diameter. Products with nanomaterials as their basis are diverse, including diagnostic and therapeutic agents, stain-resistant clothing, solar cells, sun blocks, and cosmetics, and an expanding array of applications is anticipated. The increasing production and use of engineered nanomaterials may lead to greater exposures of workers, consumers, and the environment, and raises concerns about potential harms to human and ecosystem health. This paper addresses the general topic of research on engineered nanomaterials, health, and the environment. It covers the history of research planning on engineered nanomaterials, giving emphasis to the recent reports from a committee of the US National Research Council. The two reports from this committee offered a framework-based research strategy intended to address critical uncertainties. This paper ends with general lessons learned from experience with engineered nanomaterials that may apply to other emerging environmental threats.
\end{abstract}

Keywords Nanotechnology $\cdot$ Research strategies $\cdot$ Human health $\cdot$ Environmental health and safety $\cdot$ Risk assessment

\section{Introduction}

Products incorporating engineered nanomaterials are a rapidly growing manufacturing sector, with revenues from nano-

J. M. Samet

Department of Preventive Medicine, Keck School of Medicine of

USC, Los Angeles, CA, USA

\section{J. M. Samet $(\bowtie)$}

Institute of Global Health, University of Southern California, 2001

North Soto Street, Suite 330A, Los Angeles, CA 90089-9239, USA

e-mail: jsamet@med.usc.edu enabled products increasing from $\$ 110$ billion in 2010 to \$318 billion in 2013 in the US, according to a market research study commissioned by the National Science Foundation and the National Nanotechnology Coordination Office [1]. The word 'nano' means very small, but when used with reference to size it means one billionth or $10^{-9}$. While there is an extraordinary diversity of engineered nanomaterials, they all incorporate building blocks that are initially at the nanoscale, i.e. less than 100 nanometers or $0.1 \mu \mathrm{m}$ in greatest diameter, even if the ultimate product is far larger [2•]. Products with nanomaterials as their basis are diverse, including diagnostic and therapeutic agents, stain-resistant clothing, solar cells, sun blocks, and cosmetics, and an expanding array of applications is anticipated. These varied applications reflect the unique properties of engineered nanomaterials. Commonly used nanomaterials include fullerenes, quantum dots, metal oxide nanoparticles, such as zinc oxide nanoparticles, and carbon nanotubes $[2 \cdot]$.

Given the potential for widespread application and the rise of manufacturing of engineered nanomaterials, governments have implemented initiatives to promote the growth of the nanotechnology sector. In the US, the National Nanotechnology Initiative (NNI) originated with a speech by President Clinton in the year 2000 on the potential opportunities of nanotechnology [3]. The NNI was charged with coordinating the development and application of nanotechnology and also with carrying out activities related to potential impacts of nanotechnology on the environment and human health [4]. The NNI undergoes periodic review $[5,6]$.

The same unique properties of nanomaterials that have led to their widespread application also raised concerns about potential harms to human and ecosystem health. In one widely publicized scenario, based on Michael Crichton's science fiction book Prey, self-replicating 'nanobots' threaten humanity with the possibility of forming a 'grey goo' [7]. While the idea of a 'grey goo' did not originate with Crichton, the 
emergence of nanotechnology has been construed by some as potentially posing a grave threat to the environment and to workers in nanotechnology and even to the general population. More rational discussion has been directed at how to organize research to understand whether engineered nanomaterials pose a threat to human and ecosystem health.

This paper addresses the general topic of research on engineered nanomaterials, health, and the environment. It covers the history of research planning on engineered nanomaterials, giving emphasis to strategies taken to develop multidisciplinary research strategies and the gaps in the resulting evidence. Over more than a decade, multiple research agendas on engineered nanomaterials have been elaborated through multidisciplinary stakeholder processes that have engaged researchers in academia, government and industry; non-governmental organizations; public agencies; and manufacturers. Key agendas have come from various governmental agencies within the US and elsewhere, nongovernmental organizations concerned with the environment and human health, and the US National Research Council (NRC) $[2 \bullet, 8 \bullet$. These agendas have identified critical areas of uncertainty and the research needed to address the gaps. Here, emphasis is given to the findings of the NRC's Committee to Develop a Research Strategy for Environmental, Health, and Safety Aspects of Engineered Nanomaterials, which has released two reports: the first in 2012 that set out a research agenda [2•], and the second in 2013, which considered progress and updated the prior volume's strategy [8$\cdot$.

The issues involved in developing a research agenda for potential health and environmental impacts of engineered nanomaterials are both specific and general. Substantial effort is expended on developing research agendas on various topics by such entities as the National Academy of Sciences, the National Institutes of Health, and professional organizations, as well as individual scientists. This paper ends with general lessons learned from research experience with engineered nanomaterials.

\section{Background}

With regard to potential general exposures in the environment and to people, the production, use, and disposal of engineered nanomaterials follow a complicated and variable lifecycle (Fig. 1). Figure 1 provides a generic overview of the lifecycle of an engineered nanomaterial. As depicted in the figure, production of engineered nanomaterials involves initial steps at which nanoscale precursors are used to develop primary and possibly secondary products. Across this generic lifecycle, there are various points at which release is possible and exposures to humans and the environment could take place. Early in the lifecycle, there is the potential for exposures to workers, which are likely to be at higher levels than further downstream in the lifecycle.
The initial concerns about the human health and environmental impacts of engineered nanomaterials reflected the properties of nanoscale materials and long-standing evidence indicating that inhaled nanoscale materials, so-called 'ultrafine' particles, not only cause lung injury but can translocate and undergo systemic distribution $\left[9,10^{\bullet}\right]$. The sites of particle deposition within the respiratory tract, which extends from the nose and upper airway to the alveoli, depend on the aerodynamic diameter of the particles. Those under 100 nanometers in aerodynamic diameter, the ultrafine fraction, are filtered in the upper airway, but alveolar deposition is high for those particles reaching the peripheral lung. In results potentially relevant to humans, experiments involving rodents and fish show that inhaled and deposited nanoparticles can move through the alveolar epithelium and reach the general circulation or travel along the olfactory nerve and reach the brain [11, 12]. There are other pathways for exposure of humans as well, including transdermal migration of nanoparticles in products that are placed on the skin and ingestion of contaminated liquids or foods.

There is a growing literature on how nanoparticles could cause harm to human health and the environment. A literature search on PubMed using the keywords 'nanomaterials' and 'toxicology', 'human health', or 'environmental impact' shows an exponential growth in the number of publications on these topics over the past decade, but with significantly more studies on environmental outcomes (Fig. 2). These studies draw on a variety of experimental methods, including bioassays and in vitro toxicological assays; to date, epidemiological studies have been limited to several worker populations, and epidemiological studies have yet to be carried out on the general population [13•,14-17]. The two reports of the NRC committee provide summaries of the most informative studies across research domains relevant to human and ecological health $[2 \cdot, 8]$.

\section{Research Agendas for Engineered Nanomaterials}

As the promise of nanotechnology drove product innovation and rapid commercialization, concerns about the potential risks of engineered nanomaterials followed. While some of the concern reflected the unknown and seemingly menacing possibilities raised in alarmist scenarios, such as that of Prey, there was already an understanding of the risks of fibers and of ultrafine particles that provided an a priori basis for concern about engineered nanomaterials and nanotechnology. Beginning more than a decade ago, a series of research agendas were elaborated to address the numerous uncertainties related to engineered nanomaterials (Table 1 ).

Table 1 provides a compendium of some of the most significant of these agendas, as compiled by a committee of 
Fig. 1 The lifecycle and value chain of engineered nanomaterial production, use, and disposal, and potential human and ecosystem exposures (reprinted with permission from National Academies Press [2•]).

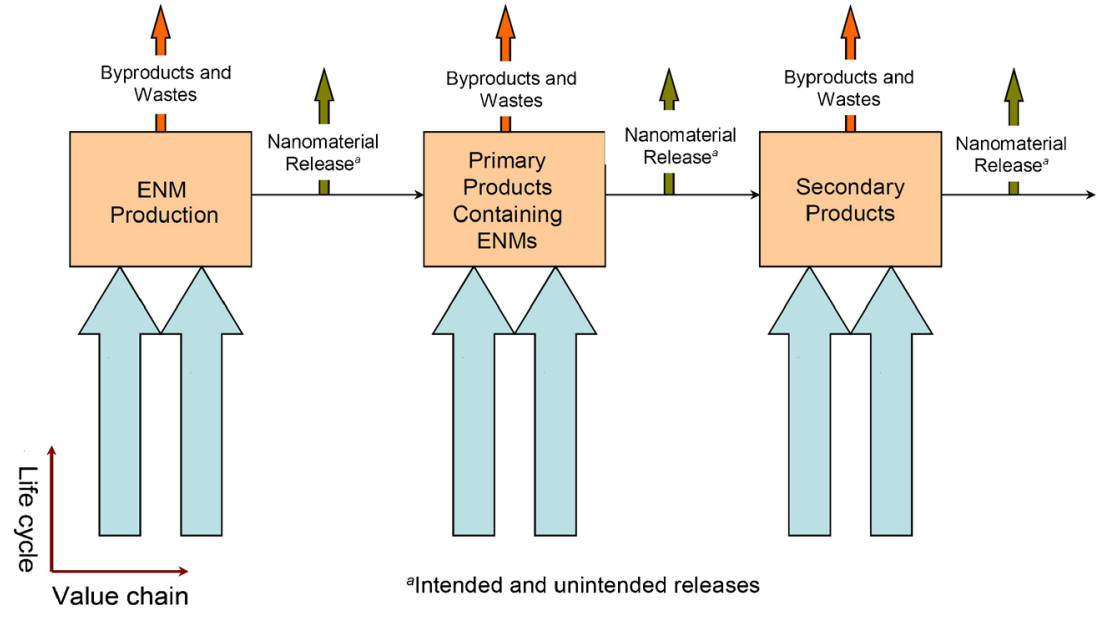

the NRC [2•]. The table is notable for the number of research agendas that have been proposed and the diverse entities that have produced them. Concern first originated in Europe. The 2004 document by the Royal Society and Royal Academy of Engineering in the UK developed one of the first notable agendas [18]. In the US, the NNI published the first interagency compilation of research needs, and further US reports came from the NNI and other agencies as well as from multistakeholder processes (Table 1) [2•]. Viewed over time, these research agendas show a growing understanding of the complexities of carrying out research on health and environmental risks of engineered nanomaterials and the need for highly multidisciplinary and integrated research strategies. The discussions underlying these agendas also led to an understanding of the overarching objective of research on engineered nanomaterials - to support the development of credible models to predict potential risks of materials to human and environmental health.
Viewed from the perspective of the NRC committee, these agendas represented a useful starting point, offering a compendium of overlapping research targets that came from differing approaches to establishing an agenda. There was also overlapping membership across the groups assembling these agendas, and these overlaps extended to members of the NRC committee.

\section{Approach of the National Research Council's Committee to Develop a Research Strategy for Environmental, Health, and Safety Aspects of Engineered Nanomaterials}

At the request of the Congress, the NRC appointed its 'Committee to Develop a Research Strategy for Environmental, Health, and Safety Aspects of Engineered Nanomaterials' (referred to here as the 'NRC committee') in 2010 with the charge of developing a strategic research agenda on potential harms to human and ecosystem health from exposures to
Fig. 2 The number of peerreviewed publications in PubMed related to nanomaterials toxicology, human health, or environmental impact from 2000 to 2013 .

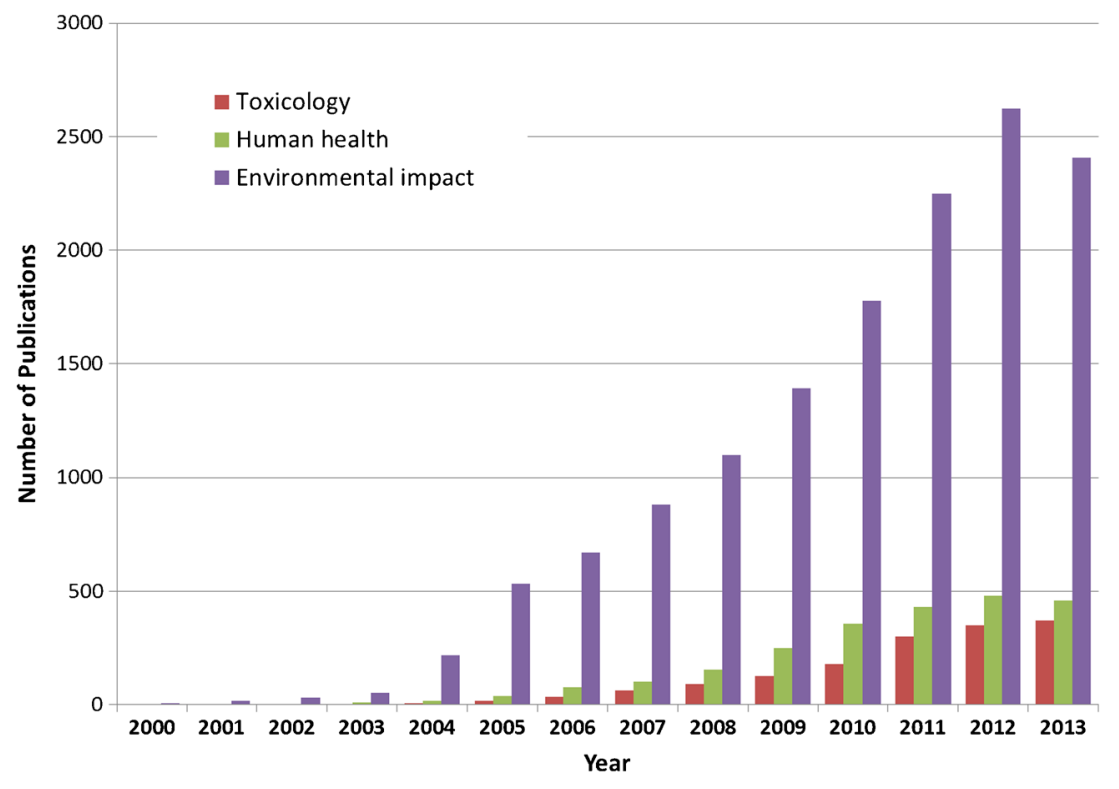




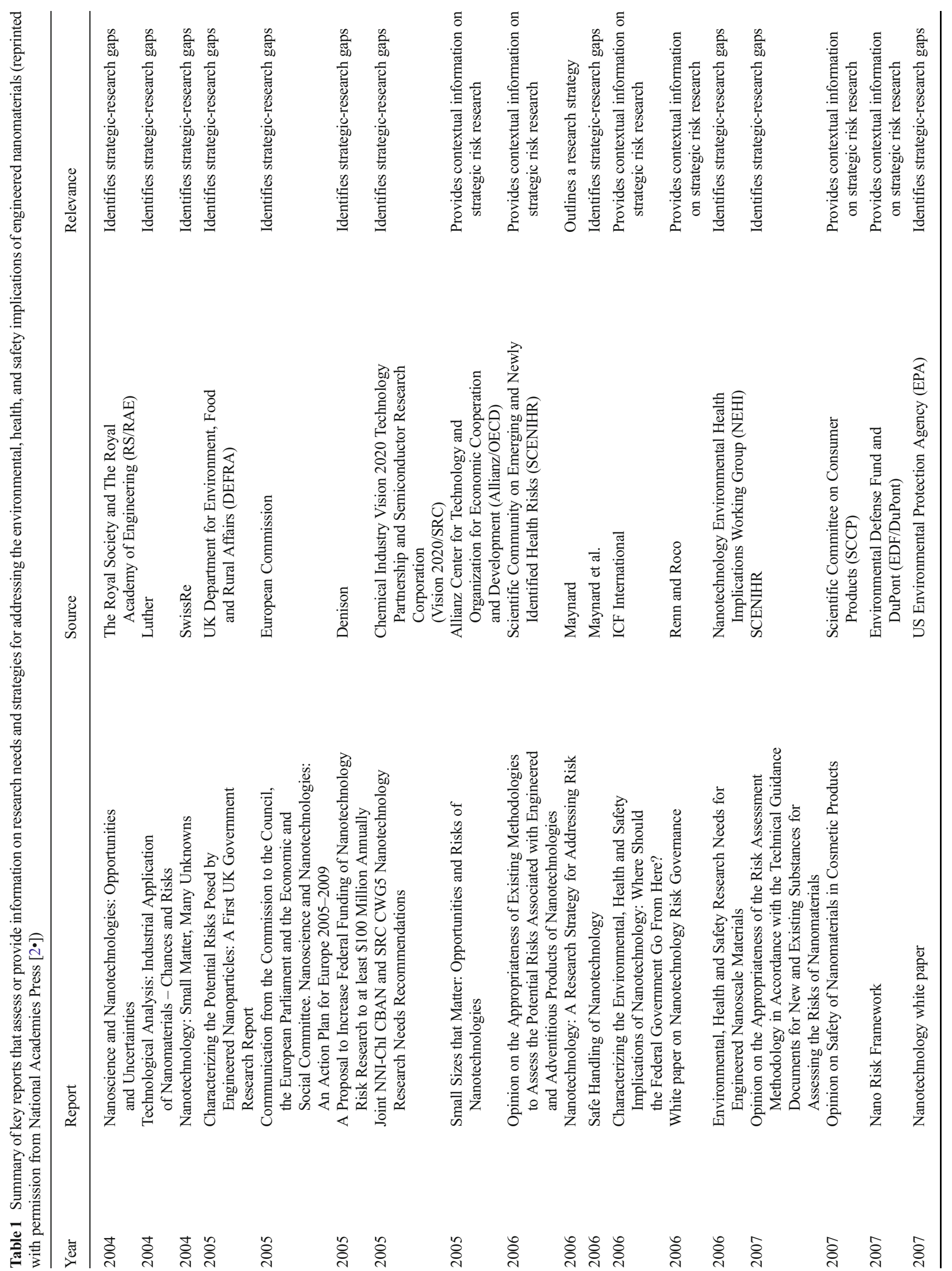




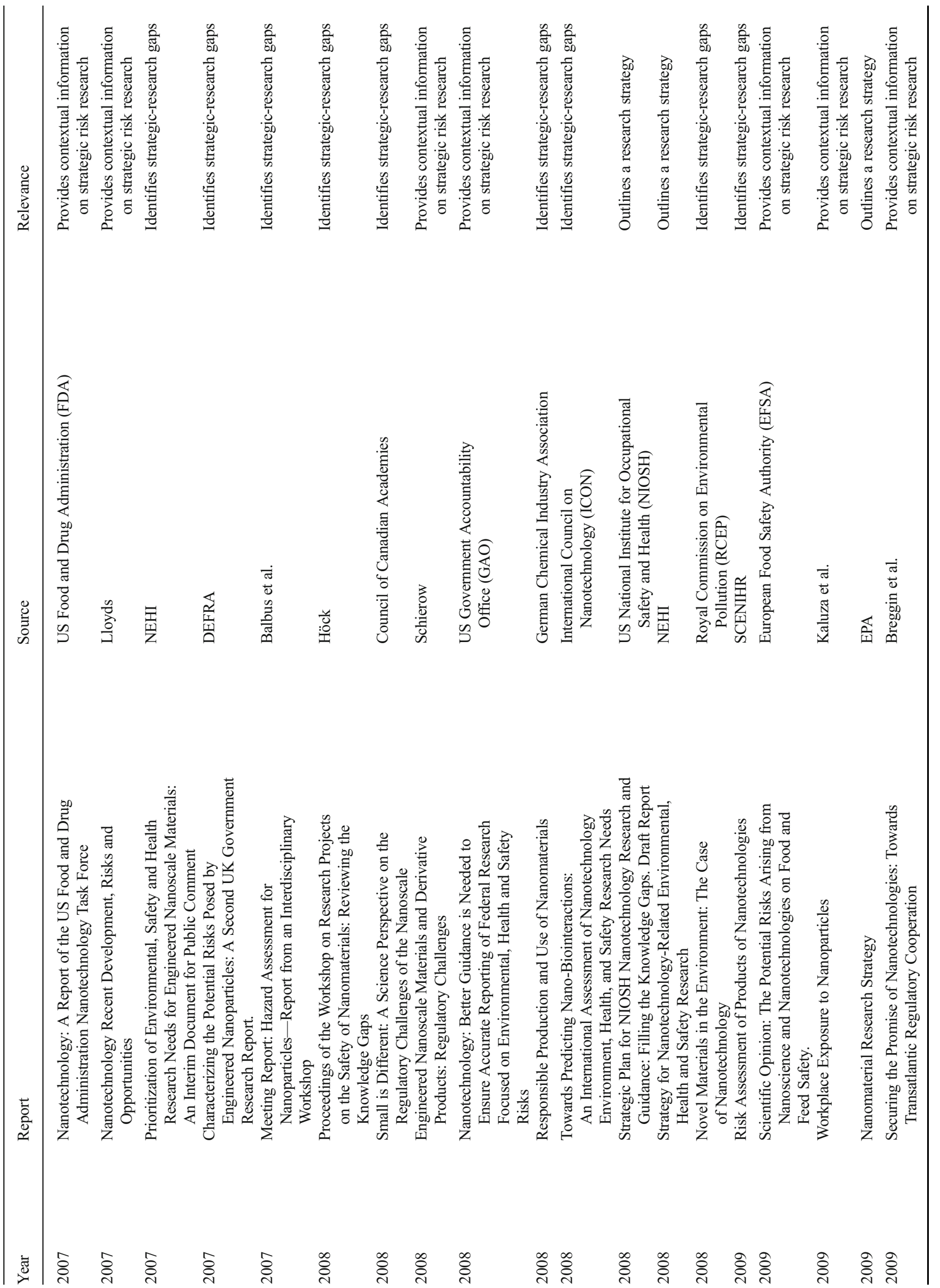



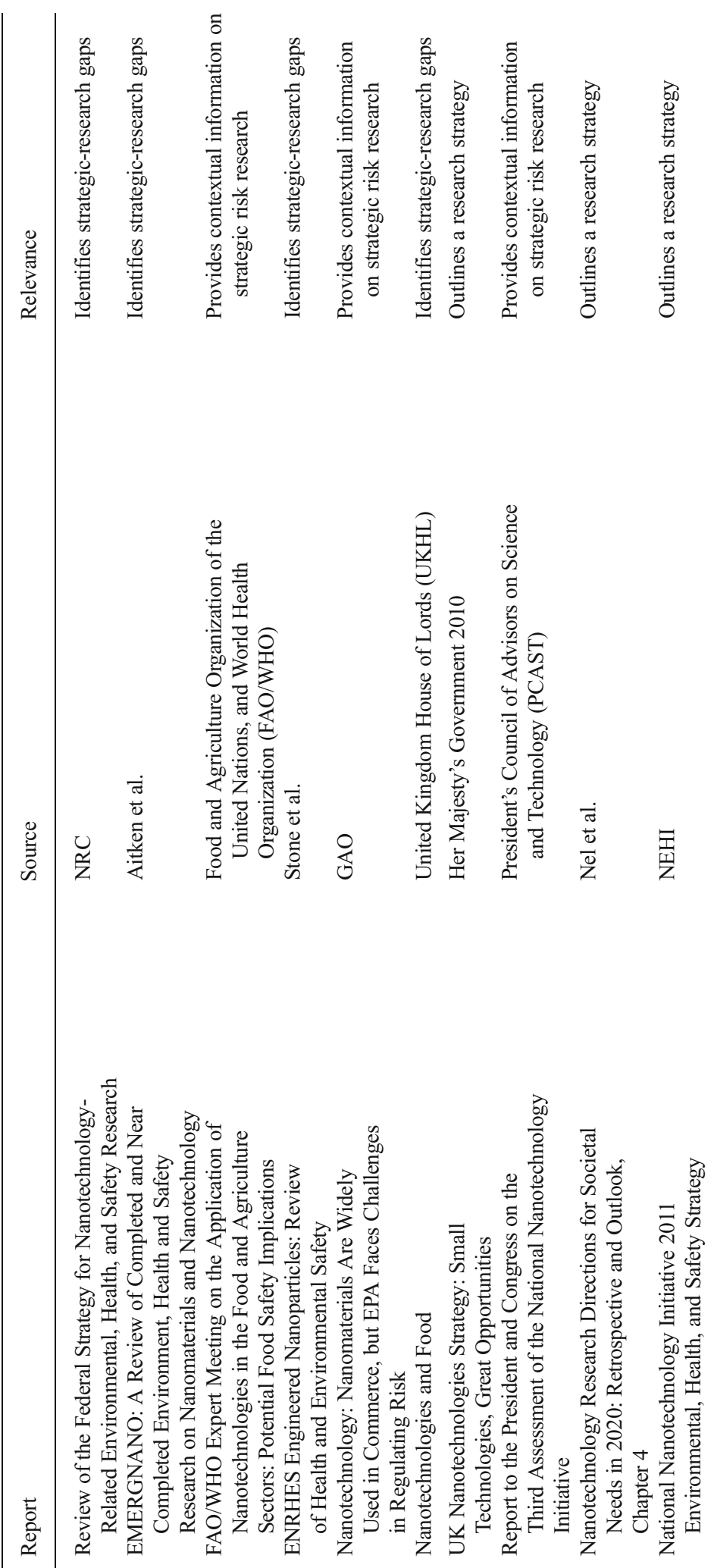

| 
engineered nanomaterials. This committee was formed in response to a $2009 \mathrm{NRC}$ report that was critical of the scope and strategy of the NNI's strategy for research on human health and the environment [19]. The committee's charge was based on a model that had been successfully applied to airborne particulate matter (PM) by the NRC's Committee on Research Priorities for Airborne Particulate Matter (NRC PM committee).

The NRC PM committee released four reports between 1998 and 2004 [20-23], the first setting out a research strategy that targeted key uncertainties and the last addressing progress towards reducing uncertainties. The agenda proposed by the PM committee was based in a toxicological paradigm that extended from sources of PM to the health consequences of PM exposure. That framework was used to identify ten critical evidence gaps with attendant uncertainties that hindered policy development and decision making. For example, a key gap was a very limited understanding of the physical and chemical characteristics of particles that determine their toxicity; that gap hindered the targeting of sources producing the particles leading to the greatest risk. The PM committee elaborated a 13-year timeline for research, estimated the associated costs on an annual basis, and offered metrics for evaluation on the short-term and long-term.

In approaching its task, the NRC committee on engineered nanomaterials first considered prior agendas (Table 1), the lifecycle and value chain of engineered nanomaterials (Fig. 1), and also developed a conceptual model for considering health and environmental impacts of nanomaterials (Fig. 3). This model, embedded in a toxicological exposureto-risk paradigm, captures the critical determinants of adverse outcomes in the central row. These determinants are not to be construed as sequential as all are critical. The circle with 'Critical Elements of Nanomaterial Interactions' refers to the biological events that result from contact of nanomaterials with biological tissues at the cellular and molecular levels. These events are considered generically as the same general interactions may be relevant to injurious responses in humans and in ecosystems.

The conceptual model also captures tools needed for research, the four pillars at the bottom of the figure. Each of the four sets of tools is indispensable: (1) standardized and characterized materials for investigation; (2) accepted and harmonized research methods; (3) models for testing hypotheses related to characteristics of engineered nanomaterials and risk, and for predicting risks of new materials; and (4) informatics for classifying materials, assays, and research results across studies to assure that data can be combined across studies.

After completing literature reviews, the committee used this model to identify critical uncertainties and the corresponding research needs, and to specify the resources required to address them. The research categories were organized around the central row of the conceptual model (Fig. 3), as follows:

- Identification, characterization and quantification of the origins of nanomaterial releases: This topic is a necessary starting point, involving ongoing inventory of engineered nanomaterials, examining the points for release, and assessing potential exposures to people and environments.

- Processes that affect both potential hazards and exposures: This broad area considers processes and macromolecular interactions that take place at scales ranging from molecular to the whole human and to ecosystems. The items included relate to bioavailability and transport
Fig. 3 Conceptual framework for informing research agenda of the National Research Council committee on engineered nanomaterials (reprinted with permission from National Academies Press [2•]).

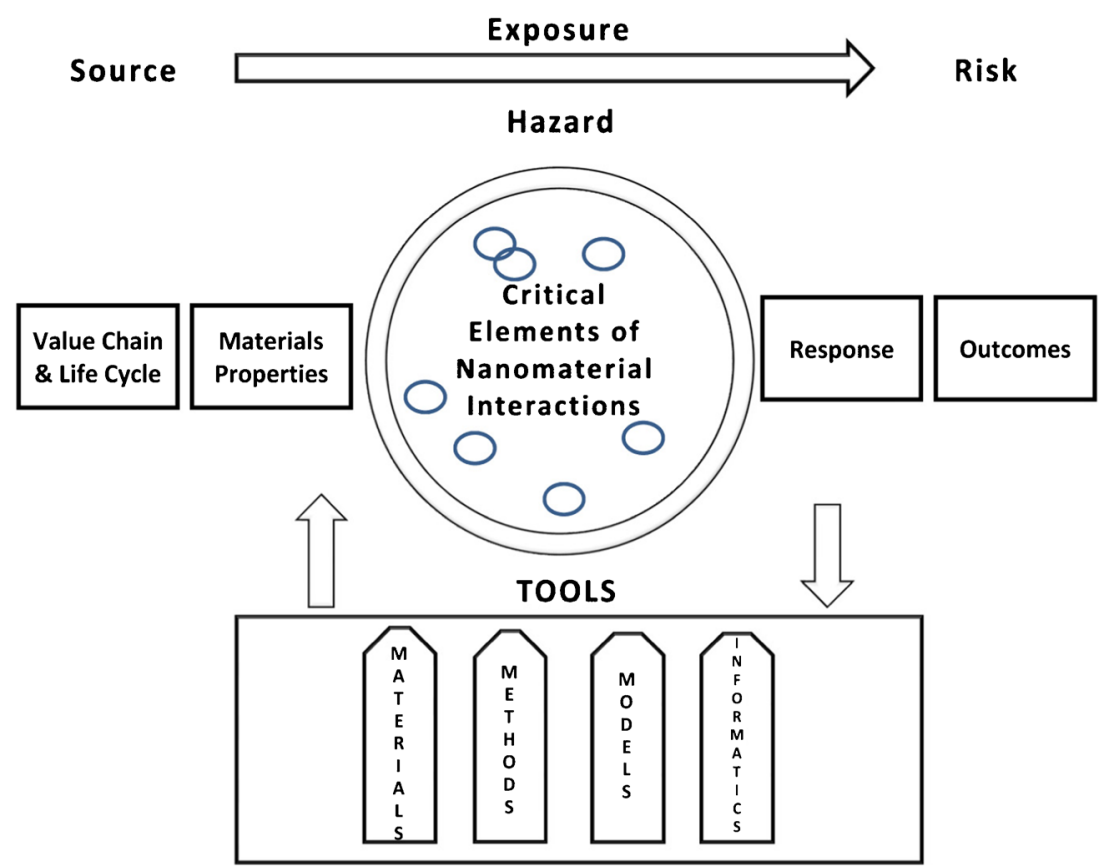


and fate, and they have the potential to affect both exposure and risk, e.g. through changes in characteristics of the material.

- Nanomaterial interactions in complex systems ranging from subcellular systems to ecosystems: This unifying topic refers to interactions of particles in a range of complex systems. It acknowledges the complexity of the processes that determine outcomes and the need for a tiered understanding that will support model development. The characteristics of the materials that determine responses are a critical focus of investigation in order to generate data for predictive models.

- Adaptive research and knowledge infrastructure for accelerating research progress and providing rapid feedback to advance research: This broad area acknowledges the need for a foundation of infrastructure to support research. Its elements correspond to the pillars of Fig. 3, which figuratively support the entire research enterprise.

The committee was also charged with considering implementation of the research agenda, the resources needed to execute it, and the metrics for evaluation of progress. These are also critical components of a research plan. With regard to the financial support needed, the NRC committee made recommendations that were constrained by the magnitude of existing funding for research on health and environmental consequences of engineered nanomaterials. The committee recognized that unrealistic suggestions would not be useful, but it did propose modestly increased funding and reallocation of resources to emphasize support of infrastructure development early in the course of its research agenda.

The NRC committee was charged with preparing a followup report that would assess progress since the first report and further refine the research agenda. Because only a short interval had elapsed since the first report, the second report largely focused on process indicators to assess the impact of the first report. To evaluate progress, the committee used a qualitative, 'traffic-light' classification of advances for each of the research topics specified in the first report. Colors were assigned, based on committee consensus, with green indicating substantial progress, yellow indicating moderate or mixed progress, and red indicating little progress. Unfortunately, progress scored poorly with 1 green, 15 yellow, and 4 red. The process of classifying progress proved to be lengthy as consensus across the committee was sought for each research priority.

Importantly, in the second report, the committee refined its vision of the fundamental approach to research on the human health and environmental risks of engineered nanomaterials (Fig. 4). This figure proposes the ideal, an integrated 'big science' research enterprise directed at potential risks of engineered nanomaterials to health and the environment. The figure diagrams the research enterprise, offering a

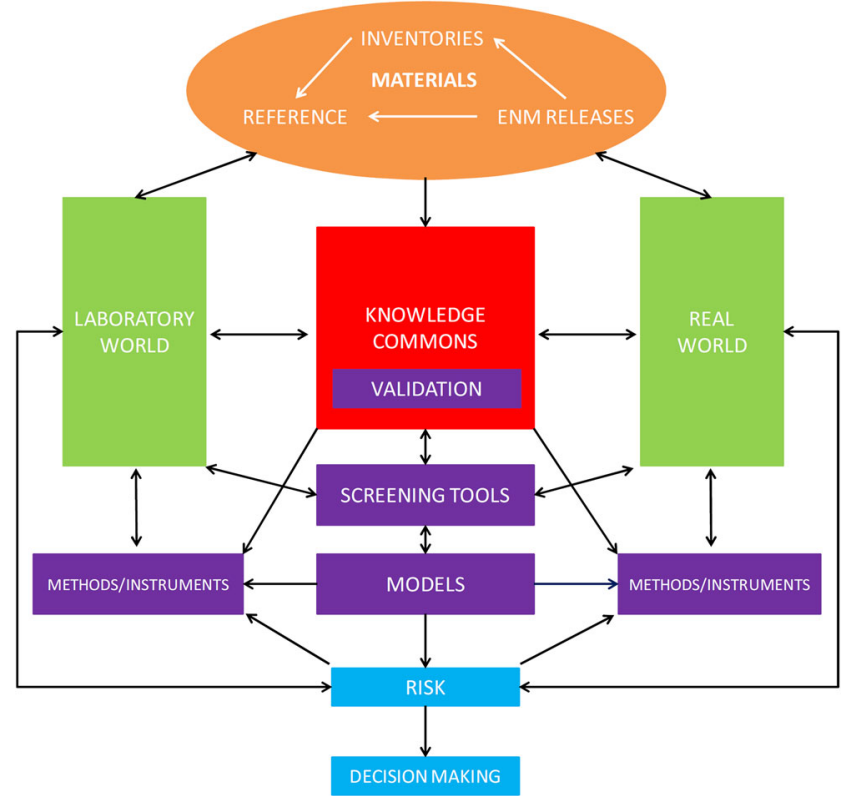

Fig. 4 Nanotechnology environmental, health, and safety research enterprise (reprinted with permission from National Academies Press [8•]).

simplistic stratification into 'laboratory world' and 'real world', referring to experimental exposures and exposures of the consequences of actual exposures for people and ecosystems. The data from these two lines of research are housed in the 'knowledge commons' which serves as a general resource for investigators and as the data foundation for modeling. The diagram also shows the path to evidence-based decision modeling. While idealized, the diagram provides a framework for how research findings from diverse lines of investigation can be brought together so that models can be developed to estimate risk and support decision making. The diagram also makes clear the central role of research infrastructure.

\section{Conclusions and Lessons Learned}

Research agendas on risks of nanomaterials have advanced for more than a decade. Their overall impact on research directions cannot be readily assessed, but there has been substantial growth of research in this area, beginning in 2004 when the first agenda was proposed (Fig. 2). In the US, the first report of the NRC committee was used by the NNI as it revised its research strategy. The NRC committee's general recommendations were echoed by the 2010 report of the President's Council of Advisors on Science and Technology (PCAST) [5].

This concluding section addresses the following 'lessons learned':

- The development of research agendas on the potential health and environmental nanomaterials was appropriately 
motivated by concerns about the uncertainties associated with this emerging technology. The research agendas were proposed on a timely basis (Table 1) and continued to evolve as questions became more specific and critical uncertainties for decision making were identified. The research agendas were proposed by a variety of groups, including governments, research agencies, and multistakeholder groups. Thus, some had 'official pathways' for reaching key decision makers around research support, while others did not.

- The groups developing the research agendas have been highly diverse, reflecting the range of scientific methods involved and the diverse stakeholders concerned with engineered nanomaterials. The NRC committee, for example, included toxicologists, ecologists, epidemiologists, public health professionals, chemical engineers, aerosol scientists, and stakeholder representatives (business and manufacturing and non-governmental organizations) [2•, 8•]. This diversity was critical for addressing the committee's task, but brings the challenges of scientific multidisciplinary and differing stakeholder views.

- The NRC PM committee proved to be remarkably effective. Its approach provided a valuable framework for the US Environmental Protection Agency and its funding recommendations were adhered to, in part because they drove the allocation of funds for research on PM by the Agency. By contrast, the NRC committee on engineered nanomaterials did not have a direct path for affecting the quantity or allocation of funding. In offering funding guidance, the NRC committee felt obligated to begin with the level of funding in place at NNI at the time it was making recommendations. A relatively modest increment was proposed, along with a reallocation of funds across topics. The committee recognized that the general area of research on environmental and human health risks of nanotechnology was under-funded, but thought that a call for a major shift in funding from development and application to research on environmental health and safety would not be met with a response.

- The NRC committee's conceptual model (Fig. 3) offers a framework for organizing and focusing research across multiple disciplines. The NRC PM committee's risk paradigm, proposed in 1998, was widely adopted and provided a common platform for considering research on the topic of airborne PM and health. Its list of research priorities, based on reducing uncertainties, was also widely used. In the future, the NRC report on engineered nanomaterials may also become a template for guiding research in this area. More generally, experience of the reports of the two committees suggest that thoughtful elaboration of conceptual models can prove useful in guiding research planning to support decision making on environmental hazards.
- The NRC committee's depiction of the ideal 'big science' research enterprise clarifies the need for infrastructure, including informatics (now referred to in this context as 'nanoinformatics'), and networks. This area has been under-funded, in spite of its central role in creating a cohesive research enterprise. Typically, such infrastructure does not receive sufficient support, in spite of having a critical role in maximizing the impact of research.

- The development of credible and validated models is a critical outcome; this goal cannot be achieved without a common nomenclature and platforms for data sharing. For example, the findings from different studies addressing potential determinants of toxicity of nanomaterials need to be pooled to test models on robust data sets. For that purpose, a common ontology for describing the test materials is needed, as well as harmonized data structures for capturing findings. The figure also shows how data from 'the laboratory world' and 'the real world' can be brought together for model refinement and cross-validation.

- In general, metrics are needed for evaluating progress; they should be directed at initial process indicators and longer-range outcomes. The approaches elaborated by the NRC PM committee proved valuable in that instance and were adapted by the NRC committee on engineered nanomaterials. Criteria were established in its first report for setting priorities - scientific value, decision-making value, and timing and feasibility [20]. In its second report, it set out criteria for addressing implementation, including interaction, integration, and accessibility [21]. Its fourth report shows how these guiding criteria were applied [23]. For the longer run, it is not clear if the NNI will monitor progress using the NRC committee's approach; a strategy for research management has been proposed without assuring long-run accountability.

This case study documents a relatively unique episode; the emergence of a sweeping new technology and efforts to prospectively assess potential hazards of that technology. Multiple research strategies have been proposed, the most recent coming from the NRC's Committee to Develop a Research Strategy for Environmental, Health, and Safety Aspects of Engineered Nanomaterials. This committee's strategy captures the elements of a 'big science' initiative and could usefully serve as a model in other domains.

\section{Compliance with Ethics Guidelines}

Conflict of Interest Jonathan M. Samet declares that he has no conflict of interest. 
Human and Animal Rights and Informed Consent This article does not contain any studies with human or animal subjects performed by any of the authors.

\section{References}

Papers of particular interest, published recently, have been highlighted as:

- Of importance

1. National Science Foundation. Market report on emerging nanotechnology now available. (http://www.nsf.gov/news/ news_summ.jsp?cntn_id $=130586 \&$ org $=\mathrm{NSF} \&$ from $=$ news $)$. (Accessed May 9 2014).

2. National Research Council. A research strategy for environmental, health and safety aspects of engineered nanomaterials. Washington: National Academies Press; 2012. This report presents a strategic approach for developing the science and research infrastructure needed to address uncertainties regarding the potential environmental health and safety risks of engineered nanomaterials.

3. National Nanotechnology Initiative. NNI Vision, Goals, and Objectives. (http://www.nano.gov/about-nni/what/vision-goals). (Accessed May 8 2014).

4. National Science and Technology Council Committee on Technology (CoT), Subcommittee on Nanoscale Science Engineering and Technology (NSET). National Nanotechnology Initiative. Environmental, health, and safety research strategy. Washington, DC, 2011.

5. President's Council of Advisors on Science and Technology, Executive Office of the President. Report to the President and Congress on the Third Assessment of the National Nanotechnology Initiative. March 12, 2010. (http://www.whitehouse.gov/sites/default/ files/microsites/ostp/pcast-nni-report.pdf). (Accessed May 12 2014).

6. National Research Council. Triennial Review of the National Nanotechnology Initiative. Washington: National Academies Press; 2013.

7. Crichton M. Prey. London: HarperCollins; 2002.

8. National Research Council. Research progress on environmental, health, and safety aspects of engineered nanomaterials. Washington: National Academies Press; 2013. This report evaluates research progress and updates research priorities and resource estimates on the basis of results of studies and emerging trends in the nanotechnology industry.

9. U.S. Environmental Protection Agency. Integrated Science Assessment for Particulate Matter (final report). Washington: U.S. Environmental Protection Agency; 2009.

10. Oberdorster G, Oberdorster E, Oberdorster J. Nanotoxicology: an emerging discipline evolving from studies of ultrafine particles.
Environ Health Perspect. 2005;113(7):823-39. This review summarizes the expanding field of nanotoxicology research with lessons learned from older biokinetic studies with nanosized particles and newer epidemiologic and toxicologic studies with airborne ultrafine particles as the basis of this field.

11. Oberdorster E. Manufactured nanomaterials (fullerenes, C60) induce oxidative stress in the brain of juvenile largemouth bass. Environ Health Perspect. 2004;112(10):1058-62.

12. Oberdorster G, Stone V, Donaldson K. Toxicology of nanoparticles: A historical perspective. Nanotoxicology. 2007;1(1):2-25.

13. Brouwer D. Exposure to manufactured nanoparticles in different workplaces. Toxicology. 2010;269(2-3):120-7. This paper reviews published workplace air measurements on manufactured nanomaterials or objects.

14. Demou E, Peter P, Hellweg S. Exposure to manufactured nanostructured particles in an industrial pilot plant. The Annals of occupational hygiene. 2008;52(8):695-706.

15. Kim B, Kim H, Yu IJ. Assessment of nanoparticle exposure in nanosilica handling process: including characteristics of nanoparticles leaking from a vacuum cleaner. Industrial health. 2014;52(2): $152-62$.

16. Koivisto AJ, Lyyranen J, Auvinen A, et al. Industrial worker exposure to airborne particles during the packing of pigment and nanoscale titanium dioxide. Inhalation toxicology. 2012;24(12): 839-49.

17. Miller A, Drake PL, Hintz P, et al. Characterizing exposures to airborne metals and nanoparticle emissions in a refinery. The Annals of occupational hygiene. 2010;54(5):504-13.

18. The Royal Society, The Royal Academy of Engineering. Nanoscience and nanotechnologies : opportunities and uncertainties. London: Royal Society; 2004.

19. National Research Council. Review of Federal Strategy for Nanotechnology-Related Environmental, Health, and Safety Research. Washington: National Academies Press; 2009.

20. National Research Council, Committee on Research Priorities for Airborne Particulate Matter. Research priorities for airborne particulate matter: I. Immediate priorities and a long-range research portfolio. Washington: National Academies Press; 1998.

21. National Research Council, Committee on Research Priorities for Airborne Particulate Matter. Research Priorities for Airborne Particulate Matter: II. Evaluating Research Progress and Updating the Portfolio. Washington: National Academy Press; 1999.

22. National Research Council, Committee on Research Priorities for Airborne Particulate Matter. Research priorities for airborne particulate matter III. Early research progress. Washington: National Academy Press; 2001.

23. National Research Council, Committee on Research Priorities for Airborne Particulate Matter. Research priorities for airborne particulate matter: IV. Continuing research progress. Washington: National Academies Press; 2004. 